\title{
An investigation into the perception of dominance from schematic faces: A study using the World-Wide Web
}

\author{
C. SENIOR, M. L. PHILLIPS, J. BARNES, and A. S. DAVID \\ Institute of Psychiatry and Kings College, London, England
}

\begin{abstract}
The World-Wide Web (WWW) is considered to be a viable tool for scientific research, and several investigators have already made use of it in their studies. Although the WWW allows researchers to access a vast subject pool, questions of reliability and validity need to be addressed before it is incorporated into mainstream research. By replicating, on the Internet, an existing study (Keating, Mazur, \& Segall, 1977) on the perception of schematic faces, we were able to conclude that experimental work carried out in this manner is not necessarily biased by the medium. One difference from previous work was the effect of a smiling versus a nonsmiling face on the perception of dominance, given an identical brow position for the two faces. This was replicated on a different occasion with different subjects, which may represent a shift in attitudes to this facial configuration since the original study was conducted. Young North American males were overrepresented in our sample, but comparison with other surveys indicates that the population sampled by the Internet is becoming more representative.
\end{abstract}

The social group has a well-defined structure with a specified role for each member (Tajfel \& Fraser, 1977). Intragroup communication can occur through a number of verbal and nonverbal sources (Argyle, 1994; Van Hooff, 1972).Within the group, the leader is often described as being the individual with the potential to influence the other members (Collins \& Raven, 1969) and, as such, often exhibits a set of stereotypical behaviors, such as controlling the group conversation through eye gaze (Argyle, 1994) and adopting a more relaxed general posture than the rest of the group (Bull, 1987). Perception of facial characteristics - in particular, eye gaze - plays a crucial part in social group dynamics (Ellsworth \& Carlsmith, 1973), personality determination (Libby \& Yaklevich, 1973), and gender perception (Brown \& Perrett, 1993; Campbell, Wallace, \& Benson, 1996).

Group leaders have facial characteristics that are unique to their position. Andrew (1963) found that primates would show a lowered brow mask when dominating or threatening, whereas, in a submissive role, primates would raise their eyebrows. Van Hooff (1967) postulated that the expressions shown by primates in certain situations were the evolutionary base for human expressions. Later studies provided support for Van Hooff's theories (Ekman \& Friesen, 1971; Ekman, Sorenson, \& Friesen, 1969). Blur-

We gratefully acknowledge the assistance of Pak Sham with the statistical analysis and of the three anonymous reviewers, who provided helpful comments on an earlier draft of this paper. C.S. is supported by the John Mackintosh Trust, Gibraltar. J.B. is supported by a UK MRC Studentship. Correspondence concerning this article should be addressed to C. Senior, Institute of Psychiatry and Kings College, School of Medicine and Dentistry, 103 Denmark Hill, London SE5 8AZ, England (e-mail: c.senior@iop.kcl.ac.uk). ton Jones (1971) observed brow positions on children's faces in Western cultures and found that, during disputes, the aggressive individuals had lowered brow positions, whereas the submissive participants in a dispute had raised eyebrows.

The analysis of brow and mouth position in the perception of dominance was investigated by Keating, Mazur, and Segall (1977). This study employed black-and-white photographs of individuals who posed with a brow-raised or brow-lowered expression, together with a smiling or a nonsmiling face. Faces with lowered eye brows were rated as being more dominant than their brow-raised counterparts. Further analysis revealed that the brow-lowered variable produced the perception of dominance more than did the mouth unsmiling factor. Keating et al. repeated the initial study with schematic faces, and the results were similar. We sought to replicate and extend these findings, using a sample recruited through the Internet.

\section{THE INTERNET}

In the past 5 years, researchers have started to employ the Internet as a means of carrying out prima facie investigations (Krantz, Ballard, \& Scher, 1997). One advantage of employing this medium is the potential subject pool. Pitkow and Recker (1994) reported 18,000 Internet accesses (referred to as hits) to their survey site in the space of 38 days, whereas Kehoe and Pitkow (1996) reported a total of 38,000 respondents to their 5-year WorldWide Web (WWW) survey. Further advantages stem from the subject pool demographics, in that subjects may be drawn from a range of nationalities and settings at minimal cost. This point is especially relevant when an investigation requires a specific type of subject population- 


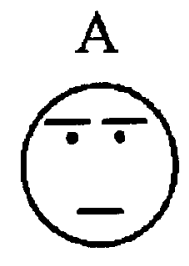

1

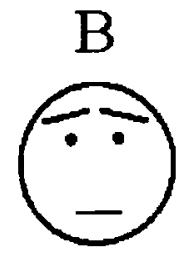

A

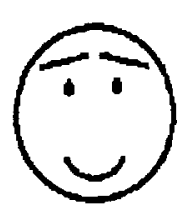

2

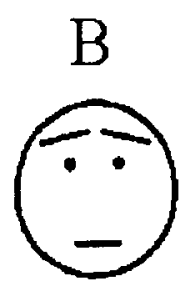

$\mathrm{B}$

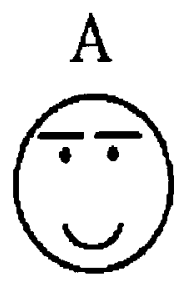

3

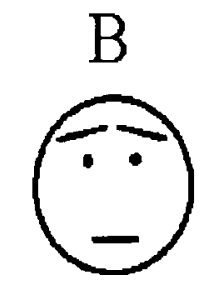

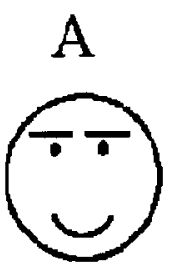

4
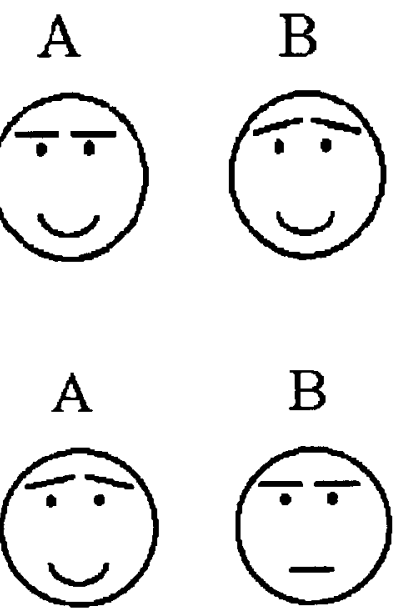

5

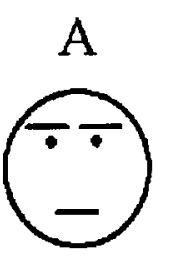

$\mathrm{B}$

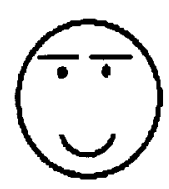

6

Figure 1. The six schematic face stimuli used in this study.

for example, individuals who are suffering from certain psychiatric conditions (Stein, 1997). There are disadvantages to using the Internet in this way. Subjects tend to be young males who, obviously, have access to computers.

Another major disadvantage is the loss of control the experimenter has over the subjects who respond to the on-line experiments. In addition, multiple users at one computer terminal, or even a single user at several computer terminals, can lead to skewed results. This can be controlled by limiting the number of responses from a single Internet address. Another disadvantage is the high drop-out rate. Studies must be short, appealing, and undemanding and must incorporate a warm-up period or an incentive to remain on line (Dickinson \& Faria, 1995).

Before the WWW can be accepted as a viable research medium, the question of its consistancy with other methods must be addressed. Clearly, the closer the results are between a study carried out on the WWW and an identical study carried out in traditional paper-and-pen manner, the more the use of the contemporary method is justified.

\section{EXPERIMENT 1}

There were two aims to this study. The first was to explore the relationship between facial configuration and the perception of social status. This was done through the manipulation of facial characteristics-eyebrow position and mouth shape - of schematic faces disseminated via the Internet. The second aim was to compare the results of this project with a previously published paper-and-pen study.

\section{Method}

Study chosen for replication on the Internet. The psychological study chosen was the schematic faces subtest described by Keating et al. (1977). This test was selected because the stimuli did not take up excessive computer memory, thus making the exercise viable, and, since they were schematic faces (see Figure 1), they were less subject to cultural biases. This is important, given the multinational subject pool we hoped to recruit.

The stimuli (simple black-and-white drawings) were chosen because they could be easily produced. Also, any difference in computer monitor technology would not affect the clarity of the images. The stimuli were made up of faces created with the graphic toolbox commands in the graphics application Paintbrush that comes as standard with the Microsoft Windows operating system and closely mimicked Keating et al.'s (1977) stimuli.

Internet site structure and execution environment. The research site was hosted on an IBM Model 95 server that ran Windows NT on EMWAC Web server software. There were no observable breakdowns of Web service throughout the month-long testing period. The WWW site constructed to support this project was written in hypertext markup language III (HTML) and consisted of eight pages. The first three were standard text pages that held instructions for the subjects and thanked them for their voluntary par- 
Table 1

Assessment of Dominance to One of a Pair of Schematic Faces in Experiment 1 and in Keating, Mazur, and Segall (1977)

\begin{tabular}{|c|c|c|c|c|c|c|c|c|c|}
\hline \multirow[b]{2}{*}{ Stimuli Number } & \multicolumn{4}{|c|}{ Experiment 1 Results } & \multicolumn{3}{|c|}{ Keating et al.'s Results } & \multirow[b]{2}{*}{ Overall $\chi^{2 \dagger}$} & \multirow[b]{2}{*}{$p$ value } \\
\hline & Left Stimulus & Right Stimulus & $\chi^{2 *}$ & $p$ value & Left Stimulus & Right Stimulus & $p$ value & & \\
\hline 1 & $106 / 112$ & $6 / 112$ & 175.9 & .001 & $112 / 115$ & $3 / 115$ & .001 & 1.13 & .29 \\
\hline 2 & $72 / 106$ & $34 / 106$ & 50.55 & .001 & $56 / 115$ & $59 / 115$ & n.s. & 8.34 & .004 \\
\hline 3 & $99 / 112$ & $13 / 112$ & 142.2 & .001 & $95 / 115$ & $20 / 115$ & .001 & 1.53 & .23 \\
\hline 4 & $101 / 112$ & $11 / 112$ & 151.3 & .001 & $104 / 115$ & $11 / 115$ & .001 & 0.004 & .95 \\
\hline 5 & $18 / 112$ & $94 / 112$ & 121.3 & .001 & $3 / 115$ & $112 / 115$ & .001 & 12.25 & .001 \\
\hline 6 & $48 / 113$ & $65 / 113$ & 53.08 & .001 & $75 / 115$ & $40 / 115$ & .01 & 11.87 & .001 \\
\hline
\end{tabular}

${ }^{*}$ Tests for equality of right and left preferences. †Tests for the homogeneity of preference in the two studies.

ticipation. The fourth page held a short demographic questionnaire that consisted of a number of check boxes, so subjects merely had to select an answer. After all of the check boxes had been selected, the on-line subject was then presented with a submit "button." This sent all of the responses to the questionnaire back to an e-mail account created for the project. After this stage, the subjects were presented with a statement that defined dominance. Shortly after this, they were presented with the actual stimuli and were asked to submit their choice of the dominant face for each of the presented pairs.

\section{Results}

The site logged 726 hits from a total of 67 different countries; $55.6 \%$ of the responses were from the United States. A total of 22 different countries were involved, with the respondents accessing the site from places as diverse as Indonesia and Russia. Of the initial respondents, only 250 individuals completed a response to the survey, and out of those, 134 were removed, owing to cases of extreme data input (e.g., entering the home country as "banana land"). The gender distribution was greatly skewed toward males $-85 \%$ of the total, as has been seen with previous surveys, although not to this extent (Pitkow \& Recker, 1994). The age distribution was approximately normal, with the 30-35 year age range being the mean. A majority of the on-line users accessed the Internet from home. Regarding educational level, the so called technology developers (i.e., those with 15-20 years of education) and the technology seekers (those with 11-14 years of education) were approximately equally represented, a pattern observed in other recent surveys (Kehoe \& Pitkow, 1996).

We analyzed the results to determine, first, the significance of the difference in proportions designating one of the two faces as dominant and, second, whether these proportions differed from Keating et al.'s (1977) study (see Table 1). Regarding this comparison, results for the first stimulus set showed no significant difference between methods. The chi-squared statistic refers to the difference in compared ratios; therefore, the null hypothesis of no differences in experimental effect is maintained. The subject responses we recorded for the second stimulus set are different, as compared with the original study $(p=.004)$. Analysis showed that the present stimulus pair number two differed in judged dominance, unlike the original study, which found no difference. The third and the fourth stimuli were judged similarly in both studies, and, thus, the null hypothesis of no differences in experimental effect cannot be rejected. The fifth and the sixth stimulus sets both showed significant statistical differences between methods. For stimulus five, the present results were in the same direction as those in Keating et al.'s study but were less strong, whereas stimulus six produced results in the opposite direction. Significantly, more of the present study sample assigned dominance to the smiling face.

\section{Discussion}

In general, the results for stimuli presented over the WWW were broadly similar to those in Keating et al.'s (1977) study, although interesting differences emerged. The data from both subject pools indicated that the straight mouth and frowned brow face was the dominant member of the presented pair. Even though the maintenance of the null hypothesis of no differences in experimental effect was not achieved in every instance, the trends were the same in four of the six pairs. Even stimulus five, which produced a weaker frown-dominance effect, still showed a significant effect in the present study. However, the final stimulus pair (number six) produced results in the opposite direction to that in Keating et al.'s. It appears that these results were produced by the effect of the mouth position in the present study. The slightly greater dominance rating for the right face in that stimulus pair goes against the "negative association between smiling and dominance" (Keating et al., 1977, p. 377). A similar pattern was seen, again, only in the Internet sample for stimulus two.

How do we explain this discrepancy with Keating et al. (1977)? There may have been a biasing element in the preamble, and it might be the case that additional instructions are needed when this type of investigation is carried out through a computer terminal. This explanation seems unlikely, however, and would be entirely post hoc, especially since, in this study (and others), frowning (lowered) brow invariably indicated dominance. It was only when the brows were raised in both faces that smiling mouth was inferred as indicating dominance. Hence, the present result could indicate a change in the judgment of attributes taken to show dominance that may have occurred in the last 20 years. Before speculating further, we attempted to replicate the results in a separate sample. 
Table 2

Assessment of Dominance to One of a Pair of Schematic Faces Presented on the Internet and in the Traditional Format in Experiment 2

\begin{tabular}{|c|c|c|c|c|c|c|c|c|c|c|}
\hline \multirow[b]{3}{*}{ Stimulus Number } & \multicolumn{4}{|c|}{ Internet Study $(n=45)$} & \multicolumn{4}{|c|}{ Paper-and-Pen Study $(n=34)$} & \multirow[b]{3}{*}{ Overall $\chi^{2+}$} & \multirow[b]{3}{*}{$p$ value } \\
\hline & \multicolumn{2}{|c|}{ Stimulus } & \multirow[b]{2}{*}{$\chi^{2^{*}}$} & \multirow[b]{2}{*}{$p$ value } & \multicolumn{2}{|c|}{ Stimulus } & \multirow[b]{2}{*}{$\chi^{2^{*}}$} & \multirow[b]{2}{*}{$p$ value } & & \\
\hline & $\overline{\text { Left }}$ & $\overline{\text { Right }}$ & & & $\overline{\text { Left }}$ & Right & & & & \\
\hline 1 & 42 & 3 & 33.9 & .001 & 34 & 0 & $\overline{\text { n.a. }}$ & n.a. & 2.356 & .13 \\
\hline 2 & 30 & 15 & 5.00 & .02 & 22 & 12 & 2.94 & .86 & 0.033 & .86 \\
\hline 3 & 36 & 9 & 16.2 & .001 & 25 & 9 & 7.42 & .006 & 0.461 & .50 \\
\hline 4 & 38 & 7 & 21.3 & .001 & 32 & 2 & 26.4 & .00 & 0.79 & .18 \\
\hline 5 & 11 & 34 & 11.8 & .0006 & 5 & 29 & 17.0 & .00 & 1.137 & .29 \\
\hline 6 & 20 & 25 & 0.55 & .45 & 15 & 19 & 0.47 & .5 & 0.001 & .98 \\
\hline
\end{tabular}

* Tests for equality of right and left preferences. †Tests for homogeneity of preference in the two studies.

\section{EXPERIMENT 2}

In Experiment 2, perception of social dominance was investigated both on the Internet and in the traditional manner, and the results were compared with those of the first experiment. The order of stimulus presentation was also varied.

\section{Method}

Stimuli. The same stimuli as those employed in the previous experiment were used in Experiment 2. In order to carry out the traditional testing, the actual picture files displayed in the WWW site were printed out, and a test booklet made up of the six face pairs was produced. The booklet's dimensions were $6 \times 8$ in., and the face pairs were presented one per page, each within a margin of approximately $2 \mathrm{in}$. The initial orienting statement that defined social dominance was printed on page one of the booklet. The laser printer resolution was high.

Procedure: Internet testing. The subject-recruiting technique was that described above, except that there were minor procedural changes that aimed to reduce drop-outs. A simple Web page was constructed with a large number of target words in the meta-heading of the HTML code. Any individual who accessed this page was invited to take part in a survey on facial recognition. The subjects were asked to read a short information statement and to submit a form with their e-mail details as indication of their consent. Upon receipt of this form, they were sent a unique password and a unique uniform resource locator (URL) of a WWW site that held the test material. The positions of each stimulus pair were randomized throughout each WWW site. The subjects were asked to submit the unique identifier with their responses for the survey; any responses that were submitted without the identifier were rejected.

Procedure: Traditional testing. The subjects were presented with the test booklet and were asked to make their responses verbally, which the experimenter noted down. After all of the responses had been made, the subjects were debriefed and thanked. The presentation of the schematic face pairs was randomized across subjects.

Subjects. There were 65 initial hits to the first WWW site, and 45 agreed to take part in the survey and submitted responses $(70 \%)$. The mean age of this subject group was 30 years (range, 1555 years), and $62 \%$ of them were male. The subjects who took part in the paper-and-pen experiment $(n=34)$ were recruited by random sampling within the staff and student population at the Institute of Psychiatry. Mean age was 32 years (range, $27-37$ years); $45 \%$ of this subject group were male.

\section{Results and Discussion}

In both the Internet and the pen-and-paper studies, the perception of dominance was significantly associated with the schematic face that had lowered eyebrows (see Table 2). Where both pairs of eyebrows were in the same position (faces 2 and 6 ), the perception of dominance appeared to favor the face that had a smiling mouth, and this finding was significant $(p<.05)$. For the subjects who were tested in the traditional manner, the responses were similar to those for the Internet group, with significance findings for four of the six face pairs. Comparison of the data from both the Internet and the traditional survey provided no significant differences.

\section{GENERAL DISCUSSION}

The data in both experiments indicate that the straight mouth and frowning eyebrow face was not always chosen as the dominant member of the pair. Although the relationship between the schematic faces of Experiment 2 in pairs two and six in the traditional test phase and pair six in the Internet test phase failed to reach statistical significance, the underlying trends were very similar to those in Experiment 1, even with the smaller subject numbers. There is, therefore, some support for the notion that the smile may be relevant to the perception of social dominance. Ethological evidence indicates that the smile in primate societies is usually an appeasement gesture (Morris, 1967; Van Hooff, 1972). Contemporary literature investigating human societies contains reports that smiling behavior is common in the facial repertoire of leaders (Butler \& Geis, 1990; Masters, Sullivan, Lanzetta, McHugo, \& Englis, 1986). This effect is demonstrated with the present British prime minister, Tony Blair, and with President Bill Clinton, who are both seen by some to be skilled proponents of the dominant smile.

Comparison of both data sets in Experiment 2 failed to reach statistical significance, and, therefore, the null hypothesis of no differences in experimental effect is maintained, and the notion that research of this type is not affected significantly by the presentation media is supported.

In order to recruit a more representative sample, future studies that utilize the Internet may find it useful to incorporate a restriction module in the HTML code that restricts the number of forms that are submitted from the United States. Another method that could be employed is the captive account technique, as used by Smith and Leigh (1997). 
Here, subjects are required to $\log$ into a remote computer account through the telnet command. The subject's details are recorded and used to prevent any action that may bias the results, such as multiple submissions to a research site. The technique described does ensure greater experimenter control, although the overall return rate is quite low. Smith and Leigh reported a total of 72 subjects in their study. In order to recruit a larger number of subjects, this study employed two different techniques.

By placing a large number of target words in the metasection of the HTML code, we managed to obtain a high initial subject response. We have called this technique passive net sampling, a device that enables an experimenter on the Internet to obtain a fairly representative subject pool-that is, one that is not constrained by a particular newsgroup. One disadvantage of this technique is lack of control, which can lead to a high drop-out rate. Once subjects realize that they are about to begin a psychological experiment, they may quickly redirect their WWW browsers to another location, or individuals may just access the WWW site, submit a number of responses, and then leave. A refinement of passive net sampling was seen with the recruiting technique employed in Experiment 2. One of the main advantages of this technique is that the drop-out rate is reduced; indeed, all the subjects who were assigned passwords submitted a complete survey. Both the present and the Smith and Leigh (1997) sampling techniques are simple devices that enable investigators with little knowledge of HTML to construct research on WWW sites.

As research based on the Internet is fairly novel, this study paid particular attention to any new ethical considerations that may have arisen. The ethical committee of the Bethlem and Maudsley Hospital trust, to which the Institute of Psychiatry is accountable, placed special emphasis on the mode of subject recruitment for this studyfor example, that those participating in the research were aware that they were doing so (see, also, Schrum, 1997).

\section{CONCLUSIONS}

This study set out to provide evidence for the validation of the Internet as a research tool by producing results similar to those of a traditional pen-and-paper study. The overall trends provide support for the Internet as a valid medium for psychological research. In this case, we have used the Internet to show the assignment of dominance to schematic faces and have demonstrated a tendency for the smile to contribute to this. A number of studies have reported similar results between Internet- and laboratorybased experiments (Krantz et al., 1997; Schiano, 1997; Smith \& Leigh, 1997). These studies do provide evidence for the growing range of variables that can be investigated on the Internet (see, also, Schmidt, 1997; Senior, Phillips, \& David, 1997). Finally, although further work is needed in order to improve the representativeness of subjects recruited via the WWW, we believe that the present study adds another step "in developing the Internet as a useful research tool" (Smith \& Leigh, 1997, p. 505).

\section{REFERENCES}

ANDREW, R. J. (1963). Evolution of facial expression. Science, 142, 1034-1041.

ARGYLE, M. (1994). The psychology of interpersonal behavior. Harmondsworth, U.K.: Penguin Books.

BLURTON JONES, N. G. (1971). Criteria for use when describing facial expressions of children. Human Biology, 43, 365-413.

Brown, E., \& Perret, D. (1993). What gives a face its gender? Perception, 22, 829-840.

Bull, P. (1987). Posture and gesture. Oxford: Pergamon.

ButLer, D., \& Geis, F. (1990). Nonverbal affect responses to male and female leaders: Implications for leadership evaluations. Journal of Personality \& Social Psychology, 58, 48-59.

Campbell, R., Wallace, S., \& Benson, P. (1996). Real men don't look down: Direction of gaze affects sex decisions on faces. Visual Cognition, 3, 393-412.

Collins, B., \& Raven, B. (1969). Group structure: Attractions, coalitions, communication and power. In G. Lindzey \& E. Aronson (Eds.), The handbook of social psychology (pp. 102-204). Reading, MA: Addison-Wesley.

Dickinson, J., \& FARIA, A. (1995). Refinements of charitable contribution incentives for mail surveys. Journal of the Market Research Society, 37, 447-453.

Ekman, P., \& Friesen, W. (1971). Constants across cultures in the face and emotion. Journal of Personality \& Social Psychology, 17, 124 129.

Ekman, P., Sorenson, E., \& Friesen, W. (1969), Pan cultural elements in facial displays of emotion. Science, 164, 86-88.

Ellsworth, P., \& Carlsmith, J. (1973). Eye contact and gaze aversion in an aggressive encounter. Journal of Personality \& Social Psychology, 28, 280-292.

Keating, C., Mazur, A., \& Segall, M. (1977). Facial gestures which influence the perception of status. Sociometry, 40, 374-378.

KeHOE, C., \& PiTKOW, J. (1996). Surveying the territory: GVUs five WWW user surveys. World Wide Web Journal [On-line serial], 1(3), 1-5. Available: http://ww.cc.gatech.edu/gvu/user_surveys/papers

Krantz, J. H., Ballard, J., \& SChER, J. (1997). Comparing the results of laboratory and World-Wide Web samples on the determinants of female attractiveness. Behavior Research Methods, Instruments, \& Computers, 29, 264-269.

LibBY, W., \& Yaklevich, D. (1973). Personality determinants of eye contact and direction of gaze aversion. Journal of Personality \& SOcial Psychology, 27, 197-206.

Masters, R., Sullivan, D., Lanzetta, J., McHugo, G., \& Englis, B. (1986). The facial displays of leaders: Toward an ethology of human politics. Journal of Social \& Biological Structure, 9, 319-343.

MORRIS, D. (1967). The naked ape. London: Cape.

PitKow, J., \& Recker, M. (1994). Using the Web as a survey tool: Results from the second WWW user survey. Journal of Computer Networks \& ISDN Systems, 27, 809-822.

SCHIANO, D. J. (1997). Convergent methodologies in cyber-psychology: A case study. Behavior Research Methods, Instruments, \& Computers, 29, 270-273.

ScHMIDT, W. C. (1997). World-Wide Web survey research: Benefits, potential problems, and solutions. Behavior Research Methods, Instruments, \& Computers, 29, 274-279.

SCHRUM, L. (1997). Ethical research in the information age: Beginning the dialog. Computers in Human Behavior, 13, 117-125.

Senior, C., Phillips, M., \& David, A. (1997). Psychiatry and the WWW: Some implications. Psychiatric Bulletin, 21, 775-778.

SmITH, M. A., \& LeIGH, B. (1997). Virtual subjects: Using the Internet as an alternative source of subjects and research environment. Behavior Research Methods, Instruments, \& Computers, 29, 496505. 
STEIN, L. (1997). Psychiatry on the Internet: Survey of an OCD mailing list. Psychiatric Bulletin, 21, 95-98.

TAJFEL, H., \& FRASER, C. (1977). Introducing social psychology. Harmondsworth, U.K.: Penguin.

VAN HoofF, J. A. R. A. M. (1967). The facial displays of the caterhine monkey and apes. In D. Morris (Ed.), Primate ethology (pp. 7-68). London: Weidenfield \& Nicholson.
VAN Hooff, J. A. R. A. M. (1972). A comparative approach to the phylogeny of laughter and smiling. In R. A. Hinde (Ed.), Non verbal communication (pp. 209-241). London: Cambridge University Press.

(Manuscript received September 26, 1997; revision accepted for publication April 17, 1998.) 